\title{
Evaluation of Acrosomal Status and Sperm Viability in Fresh and Cryopreserved Specimens by the Use of Fluorescent Peanut Agglutinin Lectin in conjunction with Hypo-osmotic Swelling Test
}

\author{
Sandro C. Esteves, Rakesh K. Sharma, Anthony J. Thomas Jr, Ashok Agarwal
}

Androfert, Center for Male Infertility, Campinas, Sao Paulo, Brazil, and Reproductive Research Center, Glickman Urological and Kidney Institute, Cleveland Clinic, Cleveland, Ohio, USA

\begin{abstract}
Objective: In this study, we evaluated whether the hypo-osmotic swelling test (HOST) can be used as a vital marker in combination with peanut agglutinin (PNA) - labeling in fresh and cryopreserved spermatozoa.

Materials and Methods: Human sperm populations were exposed to a hypo-osmotic medium for 60 minutes, and then incubated in a $1 \mu \mathrm{g} / \mathrm{mL}$ solution of the fluorescent dye Hoescht 33258 (H33258) for 10 minutes. Excess stain was removed by washing in phosphate-buffered saline (PBS) solution, and the pellet was resuspended in $100 \mu \mathrm{L}$ of culture medium. Twenty microliters of this solution were subsequently smeared on a microscope slide, and fixed in ice-cold methanol to permeabilize the sperm membranes. The fixed smears were finally incubated in a $40-\mu \mathrm{g} / \mathrm{mL}$ FITC-PNA solution for 20 minutes. Simultaneous assessment of acrosome and viability scores was done in a fluorescent microscope equipped with appropriate filters and phase contrast illumination. The same slide was examined for FITC-PNA labeling, tail swelling, and for Hoechst-33258 staining by interchanging the filters and phase contrast optics.

Results: In fresh specimens, HOST was found to provide viability assessments comparable to those obtained using the $\mathrm{H} 33258$ method $(\mathrm{r}=0.95)$. However, the results of HOST and H33258 were not correlated in cryopreserved specimens $(\mathrm{r}=$ 0.22). There was no alteration of PNA-labeling due to the HOST or H33258.

Conclusions: FITC-PNA labeling in conjunction with the visualization of the morphological change induced by exposure to hypo-osmotic solution provides a simple but effective method for establishing the state of acrosomal membrane and viability in fresh human spermatozoa, but this technique is not reliable for cryopreserved ones.
\end{abstract}

Key words: acrosome; sperm; cryopreservation; Fitc pna

Int Braz J Urol. 2007; 33: 364-76

\section{INTRODUCTION}

Specialized sperm function tests are better predictors of fertilizing potential than traditional se- men parameters assessed by standard semen analysis (1-6). Several functional tests, such as acrosome reaction, hemizona binding assay, sperm morphology, creatine kinase have been proposed to explore sperm 
fertilization ability and to predict the rate of in vitro fertilization (IVF) $(1,3,5,7)$. The acrosome reaction test $(\mathrm{AR})$ is a stable parameter of sperm function $(8,9)$ which is useful not only to predict fertilization success, but also as a tool in andrology for research purposes, such as male contraception (10) and gonadotoxic effects of food and drugs (11). The test is based on sperm physiology, and involves capacitation and acrosome reaction. Capacitation prepares the sperm to undergo the acrosome reaction with the accompanying release of lytic enzymes and exposure of membrane receptors, which are required for sperm penetration through the zona pellucida and for fusion with the oolema (12).

Several techniques have been proposed to differentiate acrosome-intact from acrosome-reacted spermatozoa, including cytochemical staining techniques $(13,14)$, indirect immunofluorescence using monoclonal antibodies (15), labeling with fluoresceinated lectins $(16,17)$ and phase-contrast microscopy to examine partial head decondensation (18). There are significant problems associated to the use of the acrosome reaction on endpoint in the evaluation of human sperm function $(14,15,17)$. One of the most important involving the diagnostic technique is the difficulty in differentiating between pathologic acrosomal loss, secondary to a reduction in cell viability, and a true acrosome loss. Although this problem may be overcome by using a DNA-sensitive fluorochromes to monitor viability, such as Hoechst-33258, the technique is labor-intensive and requires dual filter set to allow simultaneous assessment of the acrosome status and viability $(16,19)$. The differentiation between pathological and true acrosomal loss is particularly important when assessing the acrosome status of cryo-thawed spermatozoa, because cryopreservation directly damages sperm membrane, resulting in loss of membrane permeability and subsequent cell death (20), as well as in specimens with absence or limited number of motile sperm forms.

The purpose of this study was to evaluate whether the hypo-osmotic swelling test (HOST) can be used as a marker of viability in combination with fluoresceine isothiocyanate-conjugated peanut agglutinin to monitor the acrosomal status in fresh and cryopreserved spermatozoa.

\section{MATERIALS AND METHODS}

Test yolk-buffer freezing medium and sperm washing media (HEPES-modified Biggers-WhittenWhittingam) were purchased from Irvine Scientific (Santa Ana, CA). Hoechst 33258 (bis-benzimide), fluoresceine isothiocyanate-conjugated peanut agglutinin (FITC-PNA), eosin and nigrosin were obtained from Sigma Chemical Company (St. Louis, MO). Hypo-osmotic swelling solution was prepared by mixing $7.35 \mathrm{~g}$ sodium citrate and $13.51 \mathrm{~g}$ fructose in 1 liter of distilled water (21). Hoechst 33258 was prepared as a $1000 \mathrm{X}$ stock solution by dissolving 1 mg H33258 in 1 mL Dulbecoo's phosphate-buffered saline. The stock was frozen at $-20^{\circ} \mathrm{C}$ in $10 \mu \mathrm{L}$ aliquots in small foil-covered Eppendorf tubes to protect it from light.

Semen samples were obtained from 11 normal healthy volunteer individuals with proven fertility and age ranging from 21 to 35 years old (median 24). Subjects were asked to abstain from ejaculation for at least 48 hours and semen was collected by masturbation into sterile specimen cups. The ejaculate was allowed to liquefy for 30 minutes at $37^{\circ} \mathrm{C}$ and examined within 1 hour of collection to determine semen characteristics according to World Health Organization criteria (22). A computer semen-analysis of each specimen was performed on a computerassisted motion analyzer (Motion Analysis; Cell-Trak, model VP 110, Santa Rosa, CA) to assess concentration and motility. Subjects included in this study were selected according to their basic sperm parameters, as follows: volume $\geq 2.0 \mathrm{~mL}$, concentration $\geq 20 \times 10^{6} / \mathrm{mL}$ and motility $\geq 50 \%$.

Cryopreservation procedure - After initial analysis, each specimen was divided into two aliquots of identical volumes. The first aliquot was cryopreserved while the second aliquot underwent acrosomal and viability assessments. TEST yolk-buffer with glycerol was used as a freezing agent for cryopreservation. Briefly, an aliquot of the freezing medium equal to $25 \%$ of the original specimen volume was then added to the specimen. The specimen was gently mixed for 5 minutes using Hema-Tek aliquot mixer (Miles, Elkhart, Ind.). This was repeated until an equal volume of freezing medium had been added 
to the ejaculate. The specimen was then equally divided into vials for long-term cryopreservation. Cryovials were placed in the freezer at $-20^{\circ} \mathrm{C}$ for 8 minutes and thereafter in liquid nitrogen vapor at $100^{\circ} \mathrm{C}$ for 2 hours. The vials were then transferred to liquid nitrogen at $-196^{\circ} \mathrm{C}$ for long-term storage. After at least 2 days in liquid nitrogen, the vials were thawed at $37^{\circ} \mathrm{C}$ for 20 minutes, washed twice to remove the cryomedia and reconstituted in modified-BWW with $5 \%$ albumin. After thawing, acrosomal and viability assessments were performed on cryopreserved specimens.

Simultaneous assessment of acrosomal status and viability - The protocol developed for assessing the acrosome status involved the use of a detection reagent targeting the acrosomal region of the sperm head (fluorescein isothiocyanate-conjugated peanut agglutinin - FITC-PNA), in conjunction with both the hypo-osmotic swelling test (HOST) and the supravital staining Hoechst 33258 to monitor sperm viability. For this assay, $100 \mu \mathrm{L}$ of the sperm specimens were added to $1 \mathrm{~mL}$ of hypo-osmotic solution and incubated for 1 hour at $37^{\circ} \mathrm{C}$. At the end of this period, the spermatozoa were pelleted by centrifugation at 1000 rpm for 5 minutes and resuspended in $2 \mu \mathrm{g} / \mathrm{mL}$ Hoechst-33258 solution. The sperm suspensions were incubated for 10 minutes in the dark. Spermatozoa were then washed in phosphate-buffered saline (PBS) solution by centrifugation at $1200 \mathrm{rpm}$ for 5 minutes to remove excess stain, and the pellet was resuspended in $100 \mu \mathrm{L}$ of BWW. Twenty microliters of this solution were subsequently smeared on a microscope slide and allowed to dry. At least three slides of each sample were prepared, in case of problems with labeling or scoring. The slides were then immersed in ice-cold methanol for 30 seconds to permeabilize the sperm membranes and allowed to air dry. The fixed smears were immersed in a $40-\mu \mathrm{g} / \mathrm{mL}$ FITC-PNA solution, incubated at room temperature for 20 minutes in foilcovered Coplin jar, and washed gently in PBS to remove the excess label. Scoring was completed within 48 hours of staining.

A Leitz Orthoplan microscope (Leitz, Germany) equipped with phase contrast and fluorescence epi-illumination module was used to examine the slides at $1000 \mathrm{X}$ magnification in the presence of an anti-quenching agent (Cargille immersion oil, type DF, Fisher Scientific, Pittsburgh, PA) to minimize the loss of fluorescence. The same spermatozoon was first examined for tail swelling using phase contrast with halogen illumination (Figure1). Then, the illumination was changed from halogen to mercury ultraviolet epi-illumination source for assessing FITC-PNA (Figure-2) and Hoechst-33258 labeling (Figure-3). Filter cube I. 2 was used for FITCPNA, which fluoresces "apple-green" and cube A.2 for Hoechst-33258, which fluoresces a bright medium blue. Examination of the same spermatozoon for FITC-PNA labeling and for Hoechst-33258 staining was performed by interchanging the two filters. A total of 200 hundred spermatozoa per sample were scored.

Categorization of sperm tail response to hypoosmotic solution and staining patterns - Spermatozoa were classified as osmotically competent if tail swelling was observed after exposure to the hypo-osmotic solution (Figure-1). In contrary, spermatozoa were classified as osmotically incompetent if straight tail was observed. Percentage swelling induced by the cryopreservation process was determined in each specimen after thawing and subtracted from the hypoosmotic swelling test results.

Hoechst-33258 stains the nuclei of damaged cells (dead spermatozoa), which show a bright blue-

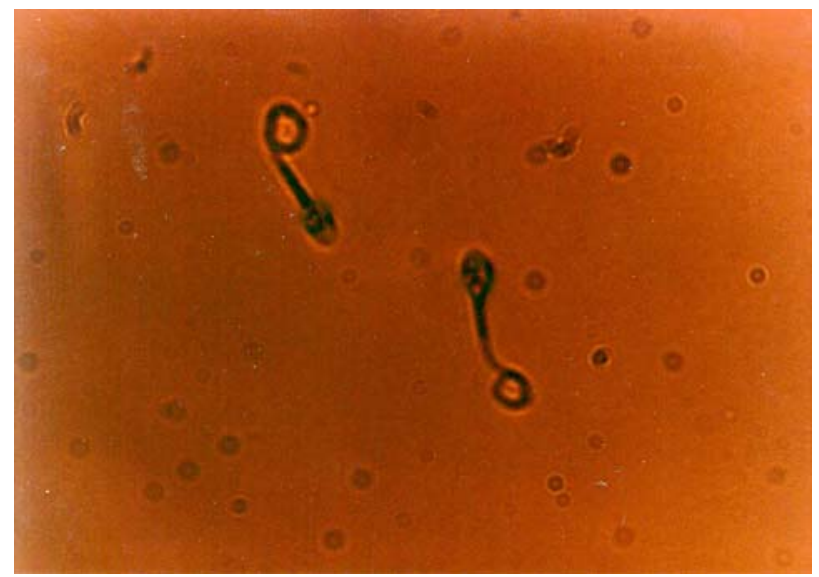

Figure 1 - Photomicrography of spermatozoa exhibiting tail swelling after exposure to the hypo-osmotic solution. Spermatozoa were examined under phase-contrast microscopy with halogen illumination at X1,000 magnification. 

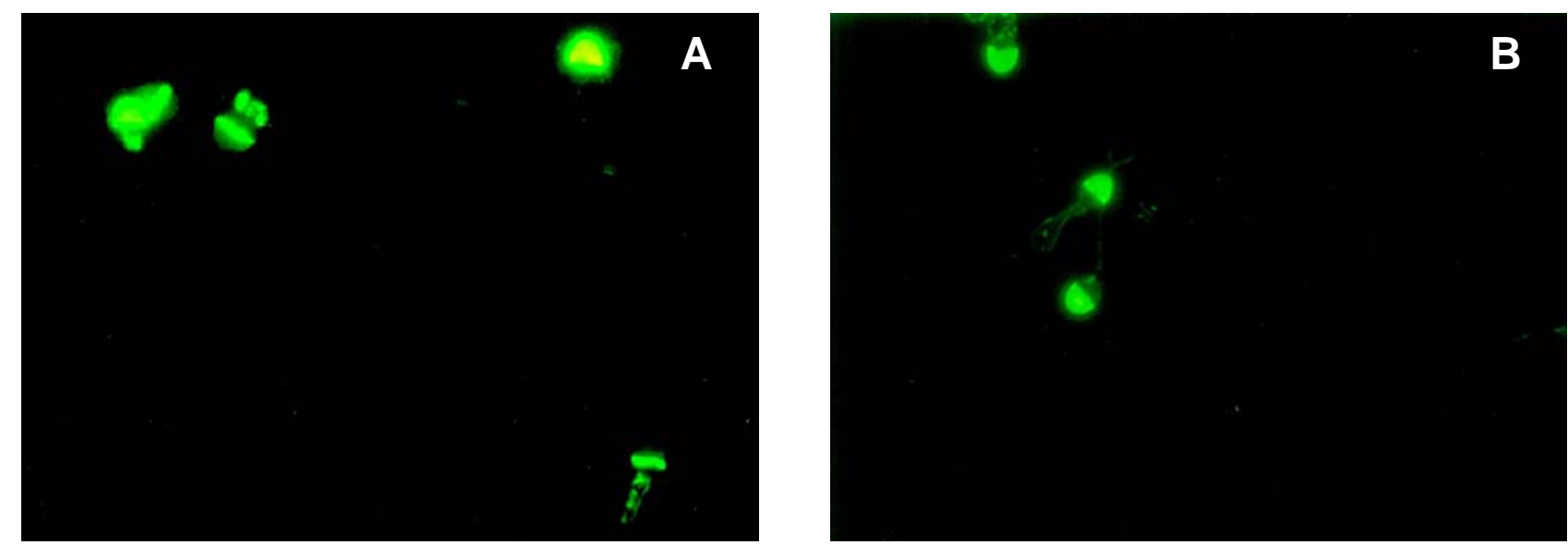

Figure 2 - Photomicrograph of spermatozoa labeled by FITC-PNA. Spermatozoa were examined under fluorescence epi-illumination at X1,000 magnification. Filter cube I.2 was used for FITC-PNA, which fluoresces "apple-green". A) In an intact acrosome, the acrosomal region of the sperm head exhibited a uniform apple-green fluorescence (left). In a reacted acrosome, only the equatorial segment of the acrosome was stained (right). B) Sperm tail swelling may be also observed if excess background staining is present.

white fluorescence and is excluded from viable cells (live spermatozoa), which show a pale blue fluorescence. Viability staining on Hoechst-33258 was classified as follows. In viable spermatozoa, the sperm head showed a pale-blue fluorescence, and in dead spermatozoa, the sperm head showed a bright bluewhite fluorescence (Figure-3) (17).

FITC-PNA binds specifically to the outer acrosomal membrane. Acrosome staining on FITCPNA labeling was classified as follows. In an intact acrosome, the acrosomal region of the sperm head exhibited a uniform apple-green fluorescence. In a reacted acrosome, only the equatorial segment of the acrosome was stained (Figure-2) (23).

Reproducibility of HOST and acrosomal evaluations - To evaluate the between-observer reproducibility of HOST, one slide from each donor was evaluated blindly by two observers. To determine the within-observer reproducibility of HOST, the same slides were re-evaluated blindly by one observer. The inter- and intra-observer reproducibility of the acrosome scores in fresh and cryopreserved specimen were previously published (24). The average coefficient of variation between observers for acrosomal results was $6.5 \%$. The intraclass correlation coefficient (ICC) between the observers was 0.81 [95\% confidence interval (CI), 0.62-0.91]. The intra-observer coefficient of variation and the ICC for acrosomal evaluations were $1.6 \%$ and 0.98 (95\% CI, 0.95-0.99), respectively.

Data are expressed as median and interquartile range. The Pearson method with an alpha level of 0.05 was used to correlate the viability results between hypoosmotic swelling test and Hoechst-33258 staining before

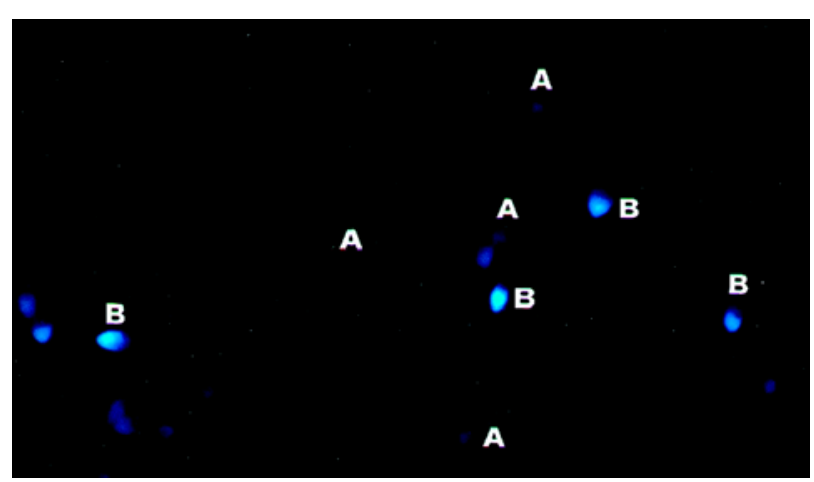

Figure 3 - Photomicrograph of spermatozoa labeled by Hoechst33258. Spermatozoa were examined under mercury ultraviolet epi-illumination at 1000X magnification. Filter cube A.2 was used for Hoechst-33258, which fluoresces a bright medium blue. Hoechst-33258 stains the nuclei of damaged cells (dead spermatozoa), which show a bright blue-white fluorescence $(B)$ and is excluded from viable cells (live spermatozoa), which show a pale blue fluorescence (A). 
freezing and after thawing. Differences in the frequency of the acrosome reaction in viable spermatozoa according to the tail swelling or Hoechst 33258 pattern were evaluated by applying the Wilcoxon signed-rank test. $\mathrm{P}<0.05$ was accepted as statistically different. Statistical analyses were performed using the SAS statistical software package (Cary, NC).

\section{RESULTS}

Sperm survival - The nuclear dye Hoechst 33258 was considered as the gold standard to determine viability. The percentage of live spermatozoa in fresh and frozen specimens was $72.0 \%(64.5 \%$ $87.0 \%$ interquartile range) and $29.5 \%(26.0 \%-36.8 \%$ interquartile range), respectively. The percentage of spermatozoa exhibiting tail swelling after exposure to the hypo-osmotic solution was $73.5 \%$ (66.0\%-85.5\% interquartile range) in fresh and 50.0\% (43.5\%-56.0\% interquartile range) in frozen specimens.

In fresh specimens, the majority of viable spermatozoa, which fluoresced pale-blue by
Hoechst-33258 staining, were also swollen by HOST (osmotically competent). The results of the HOST strongly correlated with those obtained by Hoechst33258 staining $(r=0.95, p=0.0001)$ (Figure-4). After cryopreservation, we observed no correlation between the viability scores measured by HOST and Hoechst-33258 staining $(\mathrm{r}=0.11, \mathrm{p}=0.70)$ (Figure$5)$. However, a small percentage of cryo-thawed spermatozoa were swollen before exposure to the hypo-osmotic solution (median $=20.0 \%$ [18.0\%$23.0 \%$ interquartile range]). Even after correcting the HOST results for the swelling expected from cryopreservation, the viability scores measured by HOST and Hoechst 33258 stain showed poor correlation $(\mathrm{r}=0.22 ; \mathrm{P}=0.43)$ (Figure-6).

Acrosome reaction - The evaluation of the acrosome reaction was performed by utilizing FITCPNA. Figure-7 illustrates the importance of differentiating between normally reacted cells and post mortem degeneration of the acrosome. The percentage of cells exhibiting reacted acrosomes in fresh specimens was $28.0 \%(13.0 \%-33.5 \%$ interquartile range). However, we observed a

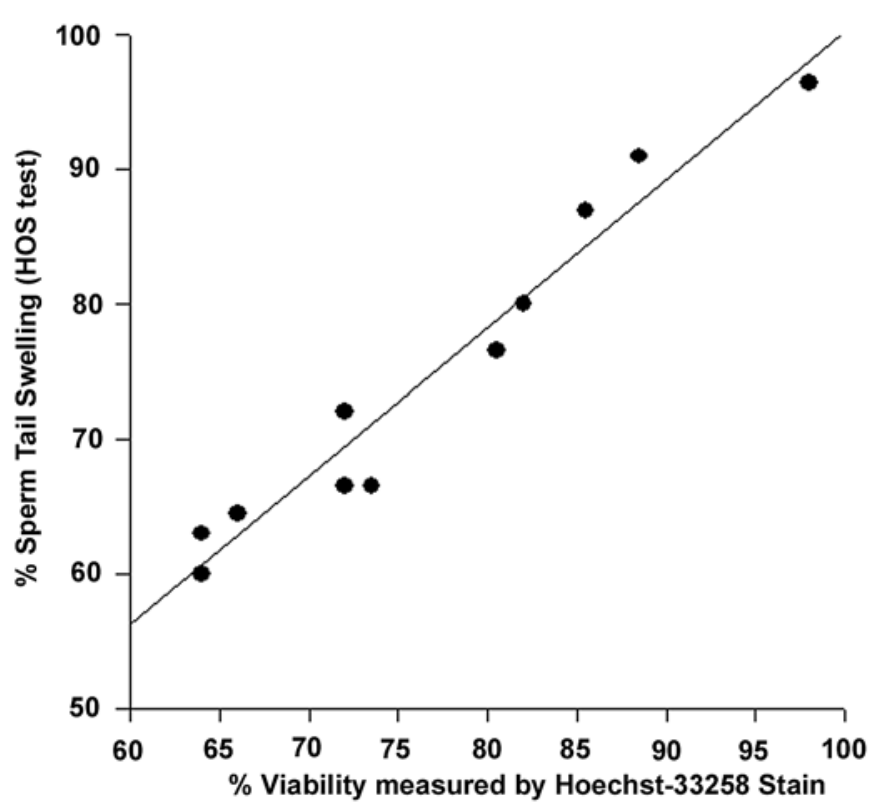

Figure 4-Correlation of viability results before cryopreservation between sperm tail swelling by HOST and Hoechst 33258. Strong correlation seen between HOST and Hoechst $33258(r=0.95, p<0.001)$. 


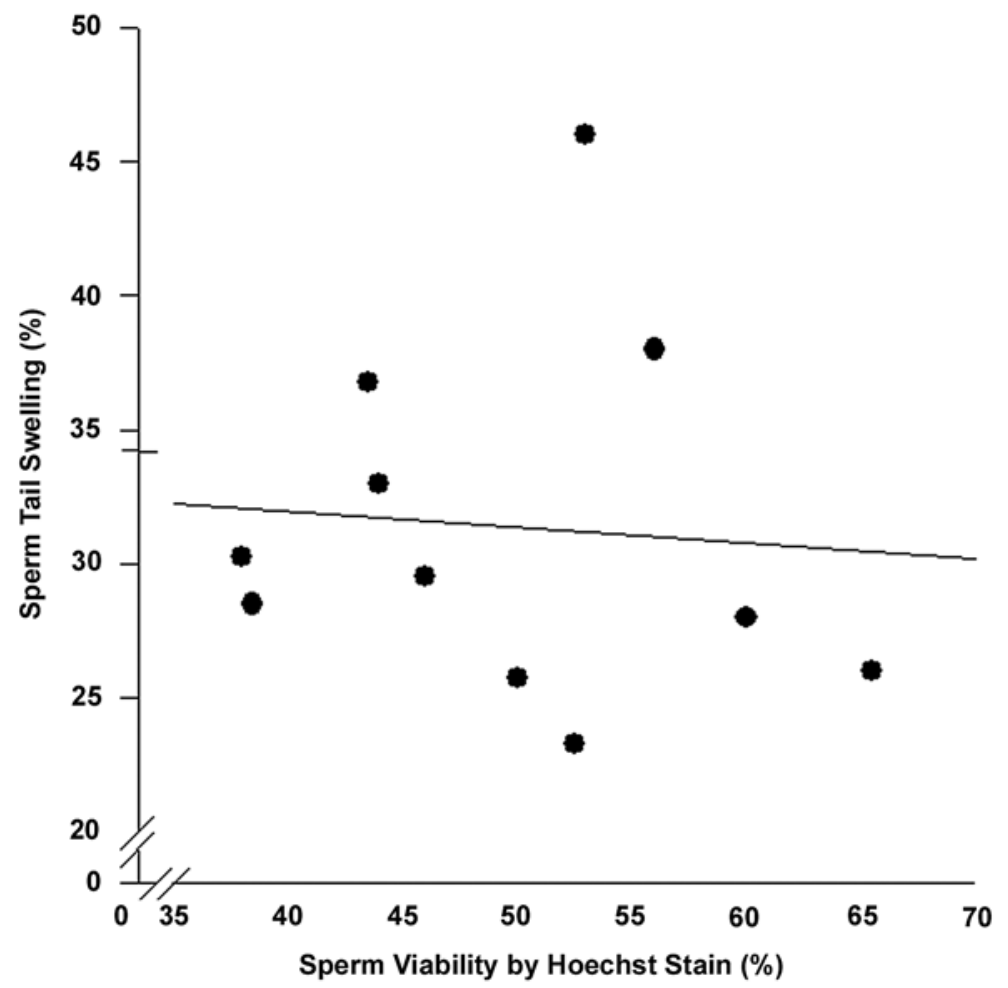

Figure 5 - Correlation of post-thaw viability results between sperm tail swelling by HOST and Hoechst 33258 stain. No correlation was seen between HOST and Hoechst $33258(r=0.11, p=0.70)$.

significant reduction in the frequency of reacted acrosomes when only viable spermatozoa, as assessed by Hoechst-33258, were evaluated (median $=7.4 \%[2.0 \%-10.9 \%$ interquartile range $] ; \mathrm{p}<0.001)$ (Figure-7).

Utilizing both methods for assessing acrosome reaction in viable human spermatozoa, (1) FITC-PNA and exposure to a hypo-osmotic solution and (2) FITCPNA combined with Hoechst-33258 stain, similar acrosome reaction rates could be detected in fresh specimens $($ median $=11.2 \% \quad[5.9 \%-15.2 \%$ interquartile range] versus $7.4 \%$ [2.0\%-10.9\% interquartile range]; $p=0.07$ ). In frozen specimens, the frequencies of acrosome reaction in viable spermatozoa assessed by FITC-Hoechst and FITCHOST were significantly different (median $=29.6 \%$ [23.1\%-33.3\% interquartile range] versus $19.5 \%$ [16.0\%-24.5\% interquartile range]; $\mathrm{p}=0.01$ ) (Figure$8)$.
The average coefficient of variation between observers for HOST was $3.5 \%$. The intraclass correlation coefficient (ICC) between the observers was 0.89 [95\% confidence interval (CI), 0.76-0.99]. The coefficient of variation for duplicate evaluation of the same slides was $1.8 \%$. The ICC between the two readings by the same observer was $0.93(95 \%$ CI, 0.89-0.99).

\section{COMMENTS}

Standard semen analysis has limited predictive value for assessing the sperm fertilizing potential (25). Functional parameters of oocytes and spermatozoa are crucial for fertilization. Oocytes must be at the proper stage of maturity. Factors in spermatozoa include motility, membrane integrity, ability to bind to the zona pellucida, acrosin activity and membrane 


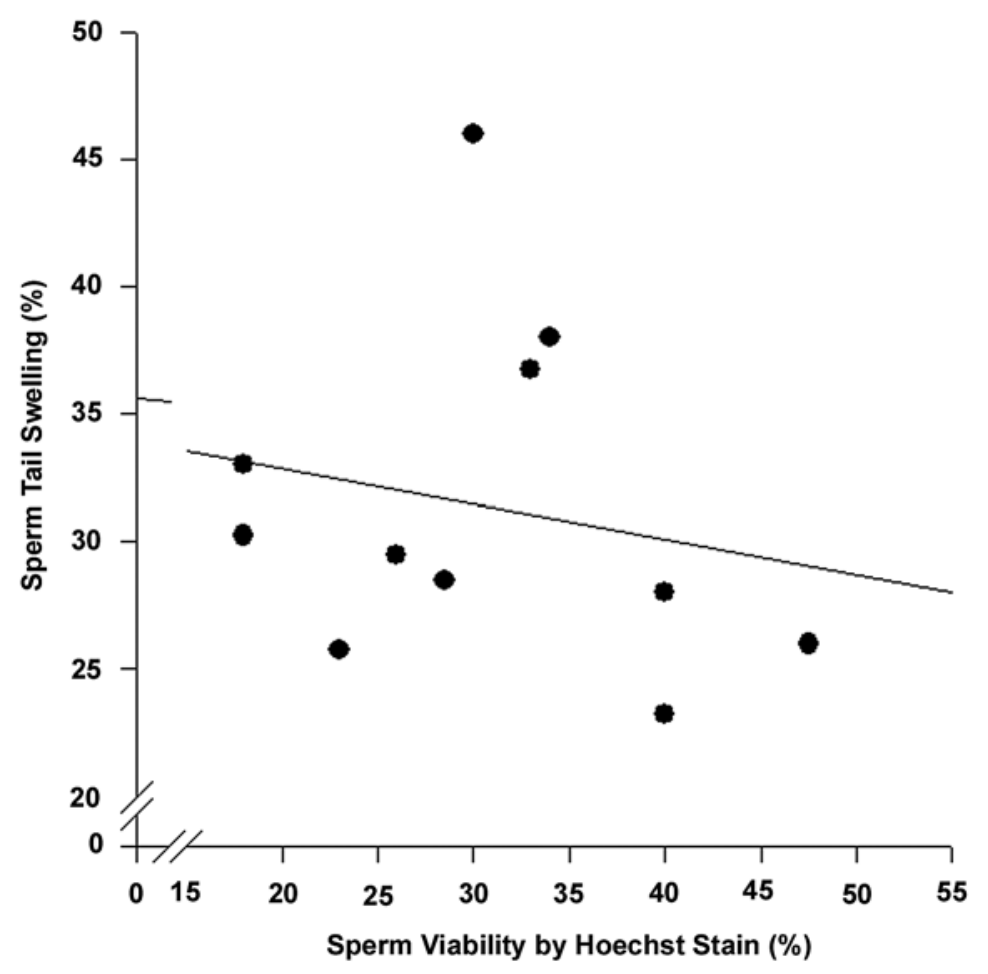

Figure 6 - Correlation of post-thaw viability results between corrected sperm tail swelling (corrected HOST) and Hoechst 33258 stain. No correlation was seen between corrected HOST and Hoechst $33258(r=0.22, p=0.43)$.

fusion ability, and also the acrosome reaction, which is of essential importance (12). Failed fertilization related to male factors, which occurs in 5-10\% of invitro fertilization cycles and 2-3\% of intracytoplasmic sperm injection cycles, may be observed in conjunction with apparent normal semen analysis $(2,5)$. For these reasons, several functional tests have been proposed to explore sperm fertilization ability and to predict the success rate of IVF. Sperm morphology evaluated by strict criteria has been shown to be relevant (26). One of the most studied in the past was the zona-free hamster egg sperm penetration assay (SPA). Although good correlation with IVF have been reported, SPA does not represent a clinical suitable test because it cannot be performed routinely, it is expensive and timeconsuming, it gives some false-negative responses, and it explores together several functions (capacitation, acrosome reaction and fusion to the oolema) (27). The ideal test system for sperm is IVF of human oocytes, which is properly governed by ethical constraints and cannot be used for a purely diagnostic purpose. Other tests such as hemizona assay, creatine kinase and the recent assays to examine sperm DNA fragmentation also seem to adequately assess the fertilizing potential of human sperm $(7,28,29)$.

Overall, most sperm function tests are laborious and expensive, and they have yet to be proved clinically relevant for routine use in clinical andrology practice. Alternatively, the acrosome reaction (AR) test, which is a simple, inexpensive laboratory test that can be performed in any andrology laboratory with no restrictions associated with the availability of expertise or the testing material, has been shown to correlate well with fertilization $(1-4,8,9)$. The AR test is based on sperm physiology. Mammalian spermatozoa must undergo a capacitation process before they are able to fertilize oocytes. Capacitation involves major biochemical and biophysical changes in the membrane 


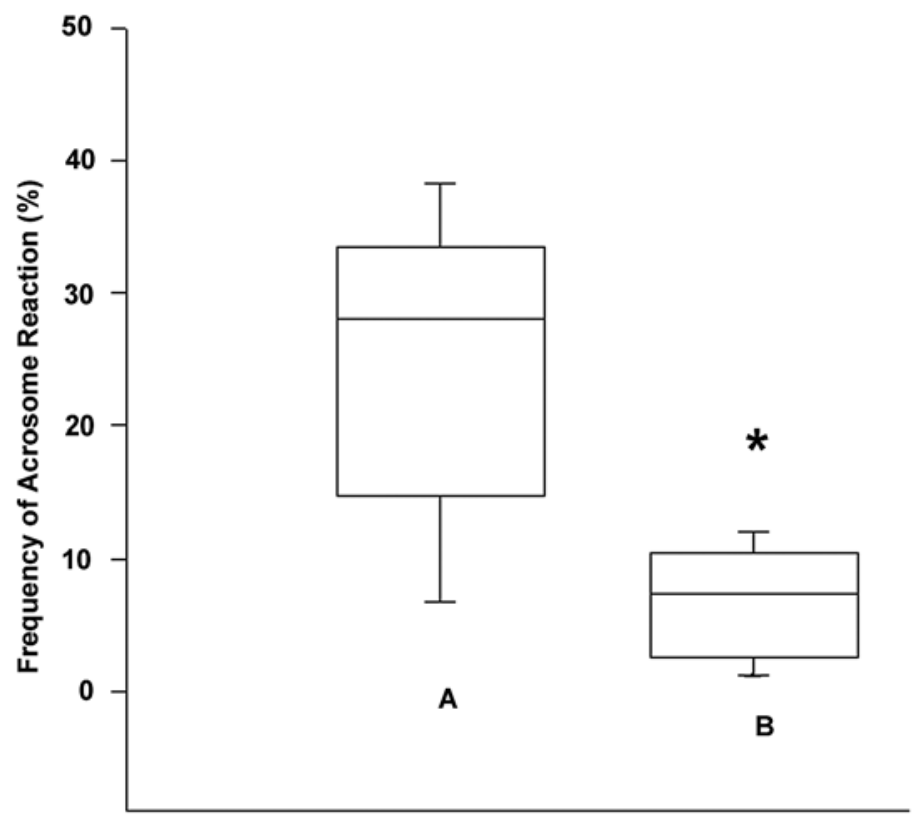

Figure 7 - Evaluation of the frequency of acrosome reaction determined by FITC-PNA and Hoechst 33258 to monitor viability from 11 samples of fertile donors. (A) Total acrosome reaction (normal reacted cells plus post mortem degeneration of the acrosomes) and (B) acrosome reaction in live human spermatozoa $(* p<0.01)$. Box covers the middle $50 \%$ of the data values, between the lower and upper quartile. The central line is the median and the whiskers extend out to $80 \%$ of the data. Bars represent values between the 5th and 95 th percentile.

complex and energy metabolism of the spermatozoa. Moreover, capacitation has been recognized as a timedependent phenomenon, with the absolute time course being species-specific. Capacitation prepares the sperm to undergo the acrosome reaction with the accompanying release of lytic enzymes and exposure of membrane receptors, which are required for sperm penetration through the zona pellucida and for fusion with the oolema (12). Integrity of acrosomal function seems to be of crucial importance to normal fertilization because acrosomeless round-headed spermatozoa cannot fertilize oocytes, and increased percentages of morphologically abnormal acrosomes were related to IVF failure. Moreover, acrosome evaluation is also a valuable tool in modern andrology for both diagnosis of male infertility and research. Premature acrosome reaction and the inability of the spermatozoa to release the acrosomal contents in response to proper stimuli (acrosome reaction insufficiency) have been associated with idiopathic male infertility (30). Additionally, the development of contraceptive vaccines involving sperm-specific antibodies that interfere in the acrosome reaction is one of the examples in the research field (10). Toxic potential of dietary substances and drugs can also be evaluated by AR assays. In a recent report, Kumi-Diaka \& Townsend investigating the toxic potential of dietary isoflavones on sperm fertility have shown that despite of the fact that light microscopic could not identify detrimental effects of phytochemicals on sperm morphology, suppression of AR in higher doses and induction of AR at lower doses have been demonstrated with AR assays (11).

Therefore, many relevant aspects can be examined by studying the sperm acrosome and determination of the acrosome reaction, such as the true potential of spermatozoa for fertilization, the search for unexplained causes of male infertility and the possibility for male contraceptive development. 


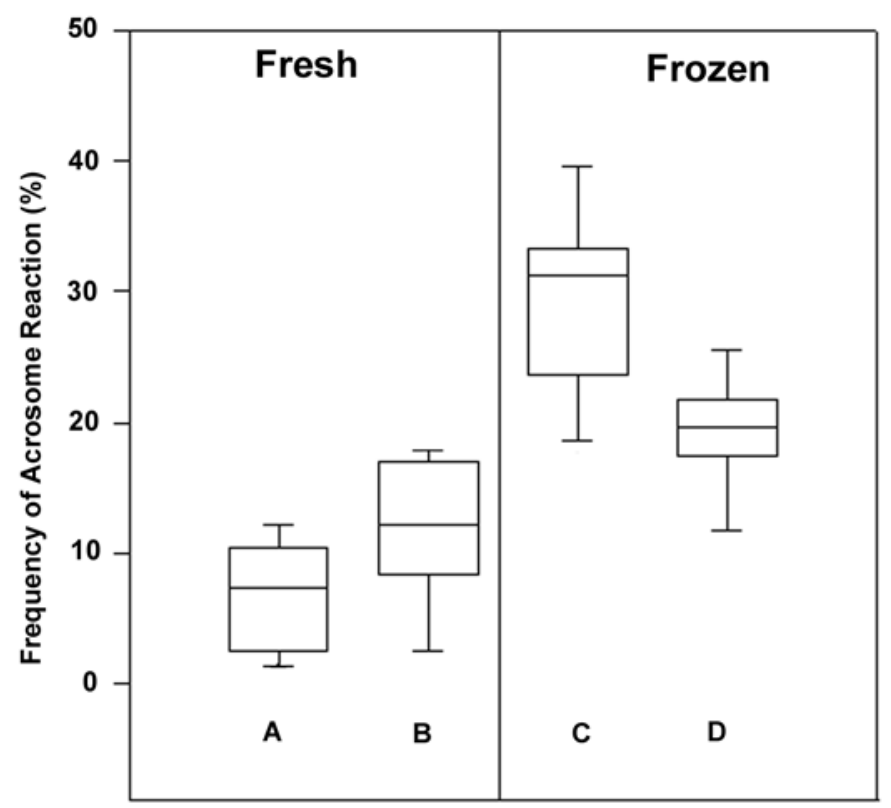

Figure 8-Evaluation of the frequency of acrosome reaction in live human spermatozoa from 11 samples of fertile donors, determined by (A) FITC-PNA and HOST, and (B) FITC-PNA and Hoechst 33258, in fresh and post-thaw specimens. Similar acrosome reaction rates were detected by both $A$ and $B$ methods in fresh specimens $(P=0.07)$. In frozen specimens, the frequencies of acrosome reaction in viable spermatozoa assessed by $A$ and $B$ methods were significantly different $(p=0.01)$. Box covers the middle $50 \%$ of the data values between the lower and upper quartile. The central line is the median and the whiskers extend out to $80 \%$ of the data. Bars represent values between the 5 th and 95 th percentile.

The human acrosome is a membrane-bound organelle, which appears during spermatogenesis as a product of the Golgi complex. It can be visualized by phase contrast light microscopy (18), and with greater accuracy by using electron microscopy, fluorescence $(1,3,16,17,19)$, monoclonal antibodies $(15)$, or simple dyes $(13,14)$. However, electron microscopy and immunofluorescence do not differentiate between normally reacted cells and post mortem degeneration of the acrosome. Cross et al. (1986) employed supravital stain Hoechst 33258 (a fluorescent DNA-binding dye with limited membrane permeability) combined with immunofluorescence technique for evaluation of acrosome reaction in viable spermatozoa (17). They observed a discrepancy between viable spermatozoa (Hoechst 33258 negative), concerning the acrosome reaction, and the total number of acrosome-reacted spermatozoa. Consequently, for exact evaluation of the acrosome reaction as a physiological process leading to fertilization, the determination of viable spermatozoa that have undergone acrosome reaction is important.

The determination of viability when studying the acrosome status in cryopreserved sperm is of fundamental importance. Cryopreservation directly damages sperm membrane, resulting in loss of membrane permeability and subsequent cell death (20). Cryopreservation can also cause permanent functional damage (sublethal damage), reducing the fertilizing ability of human sperm (19). This reduction in fertility can be explained partially by the reduction in the percentage of normal intact acrosomes and in total acrosin activity. Although damage to the acrosome after cryopreservation may also be secondary to cell death (20), an increase in the proportion of viable acrosomereacted spermatozoa after cryopreservation has been reported (19). These observations further stress the clinical importance of adequate assessment of the acrosomal status in cryopreserved sperm. 
In the present study, we developed a protocol based on Aitken et al. previous work (16) that involved sperm incubation in a hypo-osmotic solution, staining with Hoechst-33258, and staining with FITC-PNA. Hypo-osmotic swelling test (HOST) was originally developed as a clinical test for human spermatozoa (21). The percentage of swollen sperm after incubation under hypo-osmotic conditions reflect the percentage of spermatozoa with intact membranes, as it has been suggested that HOST can also assess sperm viability (31). In the present study, the viability results assessed by Hoescht-33258 and HOST were highly correlated in fresh sperm. The hypo-osmotic swelling test provided a simple, effective and convenient means of evaluating viability of spermatozoa that have been monitored for acrosome status. HOST has potential advantages over dye exclusion techniques (16). It is definitely a valuable alternative to more complex protocols involving the use of fluorochromes in fresh sperm.

On the other hand, our results fail to show correlation between viability by Hoescht-33258 and HOST in freeze-thaw sperm, even after correcting the percentage of spermatozoa swelled due to the cryopreservation process. The poor specificity of HOST in detecting viable sperm in cryopreservedthawed sperm has been previously demonstrated (31). The reasons for that are unknown, but we speculate that sperm head and tail membranes have different liabilities to the freeze-thawing process. It may be possible that the determining factor for sperm survival after cryopreservation is the membrane integrity of sperm head, which is not assessed by HOST. FITC-PNA labeling in conjunction with the HOST cannot accurately evaluate the acrosome reaction in viable cryopreserved human spermatozoa, and to date protocols involving the use of supra-vital stains have to be used in such cases.

\section{CONCLUSIONS}

Simultaneous assessment of acrosomal status using FITC-PNA labeling in conjunction with HOST provides a simple but effective method for establishing the state of acrosomal membrane and to monitor viability in fresh human spermatozoa. However, FITC-PNA labeling should not be used in conjunction with the HOST to accurately evaluate the acrosome reaction in viable cryopreserved human spermatozoa.

\section{ACKNOWLEDGEMENT}

Supported by The Cleveland Clinic Foundation Research Program Committee (grant no. 5490), Cleveland, Ohio, USA.

\section{CONFLICT OF INTEREST}

None declared.

\section{REFERENCES}

1. Katsuki T, Hara T, Ueda K, Tanaka J, Ohama K: Prediction of outcomes of assisted reproduction treatment using the calcium ionophore-induced acrosome reaction. Hum Reprod. 2005; 20: 469-75.

2. Liu DY, Baker HW: Evaluation and assessment of semen for IVF/ICSI. Asian J Androl. 2002; 4: 281-5.

3. Makkar G, Ng EH, Yeung WS, Ho PC: The significance of the ionophore-challenged acrosome reaction in the prediction of successful outcome of controlled ovarian stimulation and intrauterine insemination. Hum Reprod. 2003; 18: 534-9.

4. El-Ghobashy AA, West CR: The human sperm head: a key for successful fertilization. J Androl. 2003; 24: 2328.

5. Mahutte NG, Arici A: Failed fertilization: is it predictable? Curr Opin Obstet Gynecol. 2003; 15: 211-8.

6. Menkveld R, El-Garem Y, Schill WB, Henkel R: Relationship between human sperm morphology and acrosomal function. J Assist Reprod Genet. 2003; 20: 432-8.

7. Sidhu RS, Sharma RK, Agarwal A: Relationship between creatine kinase activity and semen characteristics in subfertile men. Int J Fertil Womens Med. 1998; 43: 192-7.

8. Calvo L, Dennison-Lagos L, Banks SM, Dorfmann A, Thorsell LP, Bustillo M, et al.: Acrosome reaction inducibility predicts fertilization success at in-vitro fertilization. Hum Reprod. 1994; 9: 1880-6. 
9. Henkel R, Muller C, Miska W, Gips H, Schill WB: Determination of the acrosome reaction in human spermatozoa is predictive of fertilization in vitro. Hum Reprod. 1993; 8: 2128-32.

10. Suri A: Contraceptive vaccines targeting sperm. Expert Opin Biol Ther. 2005; 5: 381-92.

11. Kumi-Diaka J, Townsend J: Toxic potential of dietary genistein isoflavone and beta-lapachone on capacitation and acrosome reaction of epididymal spermatozoa. J Med Food. 2003; 6: 201-8.

12. Mortimer D: From the semen to oocyte: the long route in vivo and in vitro short cut. In: Testart J, Frydman R, (ed.), Human In-Vitro Fertilization. Actual Problems and Prospects. Amsterdam: Elsevier Science Publishers. 1985, p 93.

13. Talbot P, Chacon RS: A triple-stain technique for evaluating normal acrosome reactions of human sperm. J Exp Zool. 1981; 215: 201-8.

14. Viggiano JM, Herrero MB, Martinez SP, De Gimeno MF: Analysis of the effect of nitric oxide synthase inhibition on mouse sperm employing a modified staining method for assessment of the acrosome reaction. J Androl. 1996; 17: 692-8.

15. Sanchez R, Toepfer-Petersen E, Aitken RJ, Schill WB: A new method for evaluation of the acrosome reaction in viable human spermatozoa. Andrologia. 1991; 23: 197-203.

16. Aitken RJ, Buckingham DW, Fang HG: Analysis of the responses of human spermatozoa to A23187 employing a novel technique for assessing the acrosome reaction. J Androl. 1993; 14: 132-41.

17. Cross NL, Morales P, Overstreet JW, Hanson FW: Two simple methods for detecting acrosome-reacted human sperm. Gam Res. 1986; 15: 213-26.

18. Silvestroni L, Mantovani A, Palleschi S: The partial head decondensation test is a new, quick method to assess acrosome status in human spermatozoa. Fertil Steril. 2004; 81: 1007-12.

19. Esteves SC, Sharma RK, Thomas AJ Jr, Agarwal A: Cryopreservation of human spermatozoa with pentoxifylline improves the post-thaw agonist-induced acrosome reaction rate. Hum Reprod. 1998; 13: 3384-9.

20. Cross NL, Hanks SE: Effects of cryopreservation on human sperm acrosomes. Hum Reprod. 1991; 6: 127983.

21. Jeyendran RS, Van der Ven HH, Perez-Pelaez M, Crabo BG, Zaneveld LJ: Development of an assay to assess the functional integrity of the human sperm membrane and its relationship to other semen characteristics. J Reprod Fertil. 1984; 70: 219-28.
22. WHO Laboratory Manual for the examination of Human Semen and Semen-Cervical Mucus interaction, Fourth edition. Cambridge, Cambridge University Press. 1999.

23. Mortimer D, Curtis EF, Camenzind AR: Combined use of fluorescent peanut agglutinin lectin and Hoechst 33258 to monitor the acrosomal status and vitality of human spermatozoa. Hum Reprod. 1990; 5: 99-103.

24. Esteves SC, Sharma RK, Thomas AJ Jr, Agarwal A: Effect of in vitro incubation on spontaneous acrosome reaction in fresh and cryopreserved human spermatozoa. Int J Fertil Womens Med. 1998; 43: 23542.

25. Polansky FF, Lamb EJ: Do the results of semen analysis predict future fertility? A survival analysis study. Fertil Steril. 1988; 49: 1059-65.

26. Kruger TF, Acosta AA, Simmons KF, Swanson RJ, Matta JF, Oehninger S: Predictive value of abnormal sperm morphology in in vitro fertilization. Fertil Steril. 1988; 49: 112-7.

27. Gould JE, Overstreet JW, Yanagimachi H, Yanagimachi R, Katz DF, Hanson FW: What functions of the sperm cell are measured by in vitro fertilization of zona-free hamster eggs? Fertil Steril. 1983; 40: 344-52.

28. Coddington CC, Oehninger SC, Olive DL, Franken DR, Kruger TF, Hodgen GD: Hemizona index (HZI) demonstrates excellent predictability when evaluating sperm fertilizing capacity in in vitro fertilization patients. J Androl. 1994; 15: 250-4.

29. Erenpreiss J, Spano M, Erenpreisa J, Bungum M, Giwercman A: Sperm chromatin structure and male fertility: biological and clinical aspects. Asian J Androl. 2006; 8: 11-29.

30. Tesarik J, Mendoza C: Alleviation of acrosome reaction prematurity by sperm treatment with egg yolk. Fertil Steril. 1995; 63: 153-7.

31. Esteves SC, Sharma RK, Thomas AJ Jr, Agarwal A: Suitability of the hypo-osmotic swelling test for assessing the viability of cryopreserved sperm. Fertil Steril. 1996; 66: 798-804.

Accepted after revision:

October 10, 2006 


\section{EDITORIAL COMMENT}

The authors are to be commended for their efforts to simplify the technique of concurrently assessing acrosome status (reacted vs. nonreacted) and overall sperm viability. The combination of the two methods investigated, the Fluorescein-conjugated peanut agglutinin (FITC-PNA) lectin labeling test and the Hypo-osmotic swelling test (HOST), allows for the simultaneous assessment of acrosome status and overall sperm viability, thus averting the need for dualfilter microscopy. This approach proved to be accurate for fresh sperm, but not so for cryopreserved-thawed sperm, where the HOST assay was found not to correlate with the gold

\section{EDITORIAL COMMENT}

This paper has investigated whether the hypo-osmotic swelling test and FITC-PNA allow simultaneous assessment of vitality of spermatozoa and their acrossomal status. This appears to be of particular interest since the simultaneous investigation provides evidence if acrossomal loss can be classified as physiological or degenerative. Fresh and cryopreserved samples have been used in this experimental investigation. The authors found that FITC-PNA labeling in conjunction with morphological assessment of spermatozoa after simultaneous HOST facilitates effective evaluation of acrossomal status and viability of spermatozoa in fresh samples. standard test for sperm viability, Hoeschst 33258 staining. Interestingly, the authors found that even after correcting for sperm swelling expected from cryopreservation, the viability scores measured by HOST and Hoeschst 33258 staining still poorly correlated. As the authors state, this finding suggests that the determining factor for sperm survival after cryopreservation may be the integrity of the sperm head membrane, which is not assessed by HOST. These interesting observations by the authors' make their work exciting, possibly leading to new insights into the mechanisms of sperm viability after cryopreservation and thawing.

\author{
Dr. Robert Brannigan \\ Assistant Professor of Urology \\ Northwestern University Medical School \\ Chicago, Illinois, USA \\ E-mail: R-brannigan@northwestern.edu
}

However, this technique failed to prove reliable in cryopreserved samples.

This experimental study appears to be neat and well executed, methodological flaws cannot be detected. However, the number of investigated subjects and samples has been quite small.

It should be critically mentioned that sperm function tests do not always appear superior versus conventional semen analysis. In fact these tests are usually laborious and expensive in routine analysis and mostly do not result in therapeutic consequences for the clinical andrologist. Results from this and similar studies conclusively are of some theoretical nature.

Dr. Thorsten Diemer

Klinik und Poliklinik fur Urologie

Universitatsklinikum Giessen und Marburg

Justus-Liebig-Universitat

Giessen, Germany

E-mail:Thorsten.Diemer@chiru.med.uni-giessen.de 


\section{REPLY BY THEAUTHORS}

We thank Professor Brannigan and Professor Diemer for their comments. We agree with Professor Diemer when he says that sperm function tests are usually laborious and expensive to be included in routine semen analysis. However, some of them are quite simple and inexpensive, and in general, they are better predictors of the male reproductive potential than conventional semen analysis. Additionally, sperm function tests certainly may be very helpful for the clinical andrologist. The assessment of sperm morphology by strict criteria, direct anti-sperm antibodies measurements and the tests to determine the occurrence of sperm DNA fragmentation, to cite a few, have been incorporated into the clinical practice in the recent years. These tests, which originated from the basic science, were validated as useful tools either to predict the male reproductive potential or to help the clinician define which therapeutic option is best for the infertile couple, i.e., assisted reproduction or conventional andrological treatments. 